\title{
Teses defendidas pelos candidatos no ano de 1987 nos Cursos de Pós-Graduação (CPG) na Faculdade de Direito da USP
}

\author{
DISSERTAÇŌES E TESES \\ defendidas na Faculdade de Direito em 1987
}

34. Área: Direito do Estado

Autor: Sebastiāo Botto de Barros Tojal

Dissertação: "O ESTADO E A EMPRESA ESTATAL-CONTROLE:

FISCALIZZAÇĀO OU PODER DE DOMINAÇĀO”

Orientador: Dalmo de Abreu Dallari

Defendida em: 16/6/1987

35. Área: Direito Econômico e Financeiro

Autor: José Tadeu de Chiara

Tese: "MOEDA E ORDEM JURÍDICA"

Orientador: Geraldo de Camargo Vidigal

Defendida em: 26/6/1987

36. Área: Direito do Estado

Autor: Augusto Martinez Perez

Tese: "O EXERCÍCIO DA FUNÇĀO PÚBLICA PELO CIDADĀO" Orientador: José Cretella Júnior

Defendida em: 19/8/1987

37. Área: Direito Econômico e Financeiro

Autor: Heitor Regina

Dissertação: "INCERTEZA E INSEGURANÇA JURÍDICA

RESULTANTE DO ALARGAMENTO DO PRINCÍPIO DA

LEGALIDADE NO SISTEMA TRIBUTÁRIO NACIONAL

ATRAVÉS DOS MECANISMOS DA DELEGAÇÃO E DA AUTORIZAÇÃO LEGISLATIVA"

Orientador: Walter Barbosa Corrêa

Defendida em: 20/8/1987

38. Área: Direito Civil

Autor: Custodio da Piedade Ubaldino Miranda 
Tese: "INTERPRETAÇĀO E INTEGRAÇĀO DOS NEGÓCIOS JURÍDICOS"

Orientador: Antonio Junqueira de Azevedo

Defendida em: 24/8/1987

39. Área: Direito Civil

Autor: Maria Cristina Borges de Lara Campos

Dissertação: "CONSEQÜENCIAS JURÍDICAS DA SEPARAÇÃO DE FATO NO DIREITO BRASILEIRO"

Orientador: Antonio Junqueira de Azevedo

Defendida em: 26/8/1987

40. Área: Direito do Trabalho

Autor: Antonio Rodrigues de Freitas Júnior

Dissertação: “ESTADO E REPRESENTAÇÃO SINDICAL NO BRASIL: HERANÇA COORPORATIVA E FORMAÇŌES TRIVIAIS COMO DILEMA"

Orientador: Amauri Mascaro Nascimento

Defendida em: 28/8/1987

41. Área: Direito Civil

Autor: Eliana Conceição da Silva Fernandes Machado

Dissertação: "RESPONSABILIDADE CIVIL DOS MÉDICOS"

Orientador: Fábio Maria de Mattia

Defendida em: 02/9/1987

42. Área: Direito Comercial

Autor: Antonio Martin

Tese: "CARACTERIZAÇÃO DO CONTRATO DE FORNECIMENTO"

Orientador: Mauro Brandāo Lopes

Defendida em: 29/9/1987

43. Área: Direito do Trabalho

Autor: Ênio Sandoval Peixoto

Tese: "DIREITO DO TRABALHO E INFORMÁTICA JURÍDICA"

Orientador: Amauri Mascaro Nascimento

Defendida em: 02/10/1987

44. Área: Direito do Estado

Autor: Sônia Helena Novaes Guimarães Moraes

Dissertação: “O ESTADO E A PROPRIEDADE AGRÁRIA NO BRASIL:

FÓRMULAS JURÍDICAS E VIGÊNCIA POLÍTICA"

Orientador: Dalmo de Abreu Dallari

Defendida em: 05/10/1987 
45. Área: Filosofia e Teoria Geral do Direito Autor: Ari Marcelo Solon

Dissertação: “A FUNÇĀO DO CONCEITO DE DIREITO SUBJETIVO DE PROPRIEDADE"

Orientador: Tercio Sampaio Ferraz Júnior

Defendida em: 15/10/1987

46. Área: Filosofia e Teoria Geral do Direito

Autor: Arthur Debeux Neto

Dissertação: "DIREITO E CIÊNCIA DO DIREITO (NORMATIVIDADE E INDICATIVIDADE)"

Orientador: Miguel Reale

Defendida em: 29/10/1987

47. Área: Filosofia e Teoria Geral do Direito

Autor: Mozar Costa de Oliveira

Dissertação: “O CONCEITO DA LEI NA METAFÍSICA E NA CIÊNCIA POSITIVA DO DIREITO (SANTO TOMÁS DE AQUINO E PONTES DE MIRANDA)"

Orientador: Miguel Reale

Defendida em: 05/11/1987

48. Área: Direito Penal

Autor. José Henrique Pierangelli

Dissertação: "O CONSENTIMENTO DO OFENDIDO NA TEORIA

DO DELITO"

Orientador: Paulo José da Costa Júnior

Defendida em: 05/11/1987

49. Área: Direito Comercial

Autor: Paulo Fernando Campos Salles de Toledo

Dissertaçāo: "A EMPRESA EM CRISE NO DIREITO

FRANCÊS E AMERICANO"

Orientador: Mauro Brandão Lopes

Defendida em: 06/11/1987

50. Área: Direito Civil

Autor: Lucia Stella Ramos do Lago

Tese: "SEPARAÇĀO DE FATO ENTRE CÔNJUGES-EFEITOS

PESSOAIS"

Orientador: Álvaro Villaça de Azevedo

Defendida em: 10//11/1987

51. Área: Direito Civil 
Autor: Tito Roberto Liberato

Dissertação: "RESPONSABILIDADE CIVIL DOS PAIS PELOS ATOS

DOS FILHOS MENORES"

Orientador: Carlos Alberto Bittar

Defendida em: 11/11/1987

52. Área: Direito do Estado

Autor: Massami Uyeda

Dissertação: "DA DESISTÊNCIA DA DESAPROPRIAÇÃO"

Orientador: José Cretella Júnior

Defendida em: 12/11/1987

53. Área: Direito do Estado

Autor: Sérgio Resende de Barros

Dissertação: "O VETO: UMA PROPOSTA PARA A NOVA

REPÚBLICA"

Orientador: Manoel Gonçalves Ferreira Filho

Defendida em: 27/11/1987

54. Área: Direito do Estado

Autor: Marcelo Pereira

Dissertação: "DO PODER DISCRICIONÁRIO"

Orientador: Odete Medauar

Defendida em: 04/12/1987

55. Área: Direito Civil

Autor: Antonio José de Mattos Neto

Tese: “A POSSE AGRÁRIA E SUAS IMPLICAÇÕES JURÍDICAS

NO BRASIL"

Orientador: Fábio Maria de Mattia

Defendida em: 07/12/1987

56. Área: Filosofia e Teoria Geral do Direito

Autor: Tânia Henriqueta Lotto Pierini

Dissertação: "DA BUROCRACIA À TECNOBUROCRACIA À LUZ

DE MAX WEBER"

Orientador: Tércio Sampaio Ferraz Júnior

Defendida em: 16/12/1987

57. Área: Filosofia e Teoria Geral do Direito

Autor: Celso Fernandes Campilongo

Dissertação: “REPRESENTAÇÃO POLÍTICA E ORDEM JURÍDICA:

OS DILEMAS DA DEMOCRACIA LIBERAL"

Orientador: José Eduardo Campos de Oliveira Faria

Defendida em: 18/12/1987 
58. Área: Direito Internacional Autor: Rosemea de Souza Smart

Dissertação: "ESTUDO SOBRE OS CONFLITOS DE LEIS DE ACORDO COM OS PRECENDENTES DO DIREITO NORTE-AMERICANO" Orientador: Irineu Strenger Defendida em: $14 / 4 / 1988$

59. Área: Direito Internacional Autor: Maria Luiza Machado Granziera Dissertação: “O EQUILÍBRIO INSTÁVEL: UMA COLABORAÇÃO AO ESTUDO DOS CONTRATOS INTERNACIONAIS DE FORNECIMENTO DE EQUIPAMENTO A LONGO PRAZO" Orientador: João Grandino Rodas Defendida em: 10/5/1988

60. Área: Direito do Trabalho Autor: Walkure Lopes Ribeiro da Silva Tese: “ALTERAÇĀO DO SISTEMA BRASILEIRO DE CONTROLE DA DISPENSA DO EMPREGADO"

Orientador: Amauri Mascaro Nascimento

Defendida em: 02/6/1988

61. Área: Direito do Trabalho Autor: Marcos Emanuel Canhete Dissertação: "O PRINCÍPIO DA CONTINUIDADE DO EMPREGO" Orientador: Octavio Bueno Magano. Defendida em: 07/6/1988

62. Área: Direito Civil Autor: Irany Cascone Dissertação: "ADOÇĀO E DIREITO SUCESSÓRIO" Orientador: Yussef Said Cahali Defendida em: 09/6/1988

63. Área: Direito do Estado Autor: Nelson Freire Terra Tese: "SEGURANÇA, LEI E ORDEM" Orientador: Dalmo de Abreu Dallari Defendida em: 13/6/1988

64. Área: Direito Econômico e Financeiro Autor: Roni Genicolo Garcia Tese: "MACROEMPRESA: ASPECTOS RELEVANTES PARA O DIREITO ECONÔMICO" 
Orientador: Eros Roberto Grau

Defendida em: 15/6/1988

65. Área: Direito do Estado

Autor: Mônica Herman Salem Caggiano

Tese: "SISTEMAS ELEITORAIS X REPRESENTAÇÃO POLÍTICA"

Orientador. Manoel Gonçalves Ferreira Filho

Defendida em: 16/6/1988

66. Área: Direito Civil

Autor: Francisco Antonio Paes Landim Filho

Dissertação: "O CREDOR APARENTE NO DIREITO CIVIL

BRASILEIRO (EXAME DO ARTIGO 935 DO

CÓDIGO CIVIL)"

Orientador: Fábio Maria de Mattia

Defendida em: 21/6/1988

67. Área: Direito Civil

Autor: Maria Luiza Temporini Costa Galli

Dissertaçāo: "A OBRIGAÇÃO ALIMENTAR ENTRE CÔNJUGES"

Orientador: Walter Moraes

Defendida em: 23/6/1988

68. Área: Filosofia e Teoria Geral do Direito

Autor: Cláudia de Sousa Leitāo

Dissertação: "REPRESENTAÇÃO POLÍTICA: OS DILEMAS DO

ESTADO INTERVENCIONISTA"

Orientador: José Eduardo Campos de Oliveira Faria

Defendida em: 24/6/1988

69. Área: Direito Internacional

Autor: Aquiles Augusto Varanda

Tese: “A DISCIPLINA DO 'DUMPING' DO ACORDO GERAL DE TARIFAS ADUANEIRAS E COMÉRCIO: TIPIFICAÇÃO DE UM DELITO NUM TRATADO INTERNACIONAL"

Orientador: Irineu Strenger

Defendida em: 27/6/1988

70. Área: Direito Econômico e Financeiro

Autor: Fernando Facury Scaff

Tese: "RESPONSABILIDADE DO ESTADO POR INTERVENÇĀO SOBRE O DOMÍNIO ECONÔMICO"

Orientador: Fábio Nusdeo

Defendida em: 28/6/1988 
71. Área: Filosofia e Tẹoria Geral do Direito Autor: Mário Chizzotti Filho

Dissertação: "ESTRUTURA LÓGICA DO PROCESSO JUDICIAL"

Orientador: Tércio Sampaio Ferraz Junior

Defendida em: $30 / 6 / 1988$

72. Área: Direito Internacional

Autor: Maria Cristina Lima Ribeiro Silva

Dissertação: “CONTRIBUIÇĀO AO ESTUDO DA ANTÁRTICA NO SISTEMA DAS RELAÇÕES INTERNACIONAIS"

Orientador: Vicente Marotta Rangel

Defendida em: 04/7/1988 